LBL- -33020

DE93 004708

\title{
Metallization and Insulization During Impact
}

\author{
John J. Gilman
}

Lawrence Berkeley Laboratory
University of California
Berkeley, California 94720

October 1992

This work was supported by the U.S. Department of Energy under Contract No. DE-AC03-76SF00098. 


\title{
METALLIZATION AND INSULIZATION DURING IMMPACT
}

John J. Gilman

\author{
Lawrence Berkeley Laboratory \\ One Cyclotron Road \\ Berkeley, CA 94720
}

\begin{abstract}
It is pointed out that the large strains produced by hypervelocity impacts can be expected to produce dramatic changes in the chemical bonding (electronic structures) of materials. This will change the mechanical behavior towards increased ductility when a semi-conductor is compressed until it becomes metallic; and towards increased brittleness when a transition metal is expanded so as to localize its $d$-band electrons. Both isotropic compression (expan-

sion) and shear strains can cause these transformations. Critical deformation criteria are given based on the obseived cubic to tetragonal transformations in compressed semiconductors.
\end{abstract}

\section{INTRODUCTION}

Ideal impact deformations begin with uniaxial compression. As shown in Figure $i$, this consists of a combination of a volume change (isotropic compres-sion) and a shearing distortion. In plastic materials, if the yield stress is exceeded, the stresses causing the shear distortion will tend to relax. This requires a finite amount of time which depends inversely on the difference between the local applied stress and the yield stress. The relaxation time is finite because the process requires dislocations which have finite inertial masses to be generated, multiplied, and moved.
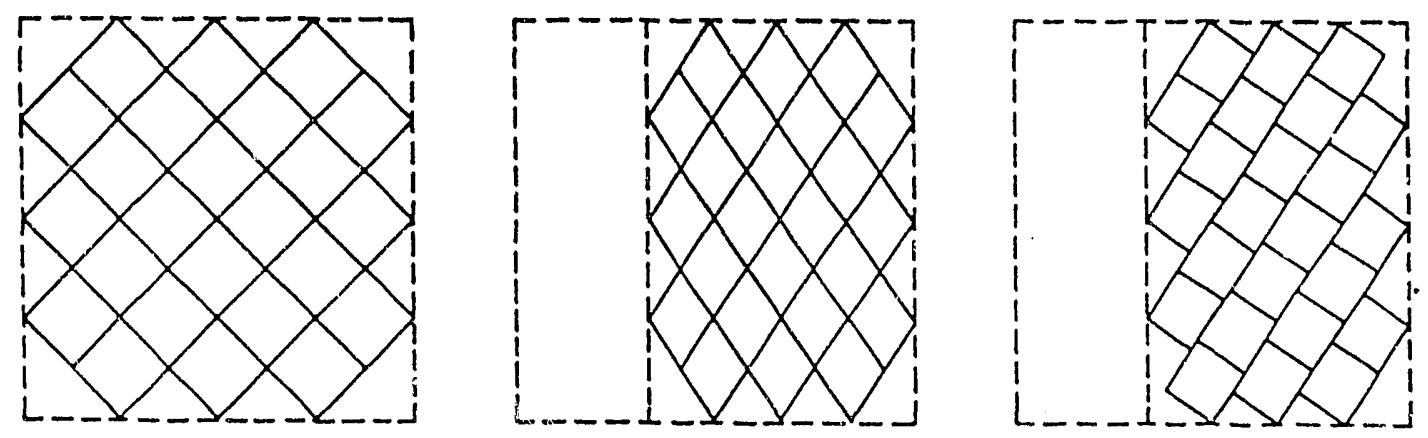

Figure 1 - Uniaxial shock compression induces both dilatation and shear in an impacted tarcet. Left - target before impact. Middle - target compressed uniaxially (deformation consists of a combination of volume reduction and shear). ' Right - Shear strains relaxed, only volume change remains.

As a result of the interaction of impact-generated compression waves with free surfaces and/or other interfaces, large amplitude rarefactions may develop. These will put the material into a state of uniaxial expansion. The deformation consists of a combination of isotropic expansion and shear, but with the signs reversed. In strong impacts the strains in the compressions and 
expansions are large, so shock fronts develop in the compressive case and instabilities (spalls) develop in the expansion case.

The local strains may far exceed the usual elastic range. This is illustrated by the schematic shock fror.t of Fig. 2 where the deformation starts at zero on one side of the front passes through an indefinitely large maximum at the front and then becomes $\Delta \mathrm{l} / \mathrm{l}=1 / 2$ on the other side. Such large strains can be expected (in some cases) not only to distort the pattern of atoms in a material, but also to cause enough distortion of the pattern of electrons (bonds) to induce electronic phase transitions. These transitions will in turn cause changes of the mechanical behavior. Note that even when the shear strains relax quickly behind the front they are very large within it.

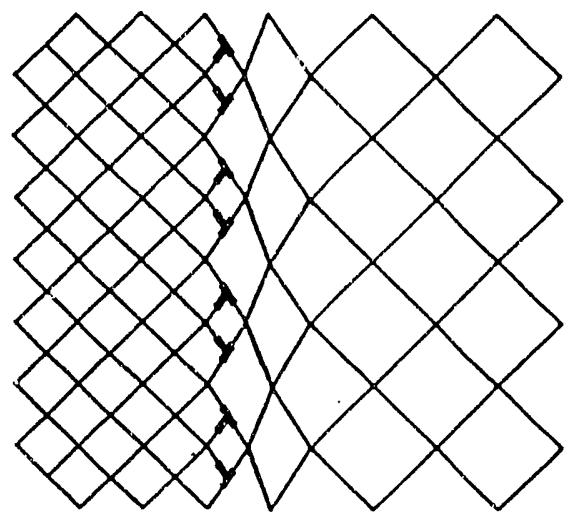

Figure 2 - Sharp shock front moving from left to right. The structural pattern is the same before and after, but at the front there are large shear distortions and a high concentration of broken "bonds" (that is, of interface dislocations).

\section{STRAIN-INDUCED TRANSFORMATIONS}

It will be shown that both compressions and shears can induce transforma-tions. Both crystal structures and transport types (semiconduction to metallic conduction and inversely) may change. Specific (albeit approximate) criteria for these transitions will be given, and compared with the available experimental data.

After an electronic transformation has occurred the material may become more ductile in the compression case; or more brittle in the tension case. Thus these transformation are relevant to hypervelocity impact phenomena because they can dramatically change the flow and fracture behavior. As an example, silicon which is normally as brittle as window glass becomes as ductile as a soft metal like lead. On the other hand a very ductile metal like copper when subjected to a strong rarefaction may exhibit cleavage-like fracture.

In the compression case (metallization), the change in mechanical proper-ties results from delocalization of the valence electrons. This enharices dislocation motion in the material by allowing the dislocation cores to spread out along the glide-planes. Prior to the transformation the atoms of the material are connected by very localized covalent bonds which localize and immobilize the dislocation cores.

In the expansion case, the s-band electrons of a metal are not expected to be affected much, but the electrons in the $d$-bands may become more localized. This may inhibit the motions of dislocations which may make the material more brittle.

Both of these effects mean that the properties of the material in the highly strained state may 
be substantially different from those in the unstrained state. Furthermore, they may not be directly deducible from a knowledge of the initial state of the material. In some cases they must play a significant role in the behavior of materials that are subjected to hypervelocity impacts.

All materials become metallic at sufficiently large compressions. However, the transformation strains are smaller for semiconductors than for large band-gap insulators; so this discussion will be limited to the former.

\section{COVALENTLY BONDED MATERIALS}

The most straightforward case is that of covalent bonding. This type yields open crystal structures (the diamond structure is the prototype), and to chemical bonds with distinct lengths and bond angles. Changing the lengths or the angles causes increases in the energies of the bonding orbitals, and decreases in the energies of the anti-bonding orbitals. In the language of solid-state physics, the valence band-edge increases in energy, while the conduction bandedge decreases. Thus the energy gap decreases (for large strains; in some cases it increases for small strains). When it vanishes, the electrons at the top of the valence band become delocalized, and the material is said to have metallized.

In general both dilating and shearing are asymmetric. Lengthening a bond is clearly not the same as shortening one. Shearing may be symmetric, but often it is not. For example, increasing a right angle making it obtuse is not the same as making it acute by decreasing it. On the other hand increasing, or decreasing, a $180^{\circ}$ angle is symmetric. Thus, depending on the sense of a particular deformation, the electrons may become either more, or less, localized.

It is well-known that insulators become metallic if they are compressed a critical amount 'Cottrell, 1988). Volumetric compression criteria for this transition have been proposed by various authors, starting with Herzfeld (1927); and later by Mott (1949); and further developed by Edwards and Sienko (1983). The proposed criteria which are well-corroborated by experiments are based on changes in the overlapping of atomic wave-functions as compression occurs. That is, on changes in bond-lengths. However, in the case of semiconductors, there is substantial experimental evidence that bond-angle changes are more important than length changes. In open structures, either bond-length or angle changes can cause the overall volume changes that have been reported as experimental results.

One pertinent fact is that uniaxial compression (a combination of dilatation and shear strains) induces the transition to the metallic state at much lower stresses than those required for triaxial compression. This has been noted in passing by various authors, but largely ignored. In the case of silicon the difference can be $40 \%$ or more, so the effect is not small (Gupta and Ruoff, 1980). Further evidence is providen by the crystallographic data to be presented here.

\section{COVALENT ELEMENTS}

A prototype transformation for semiconductors is the conversion of the diamond-framework, tetrahedrally-bonded crystals (Group IV, III-V, and II-VI substances). For eight of the known cases this converts the cubic diamond-framework into the tetragonal $\beta$-tin framework (Fig. 3). In the latter structure, the crystals are metallic (for tin itself the resistivity is not isotropic, being different parallel and perpendicular to the tetragonal axis).

The crystallographic data (Table I) show that the observed shear deformations of the bonds, $\Delta \theta / \theta$ are numerically much larger than the bond compressions $-\Delta \mathrm{l} / \mathrm{l}$. Thus, the bond-angle changes are much larger than the bond-length changes. The angle changes reduce the symmetry from cubic to tetragonal. These crystallographic facts indicate that although these 

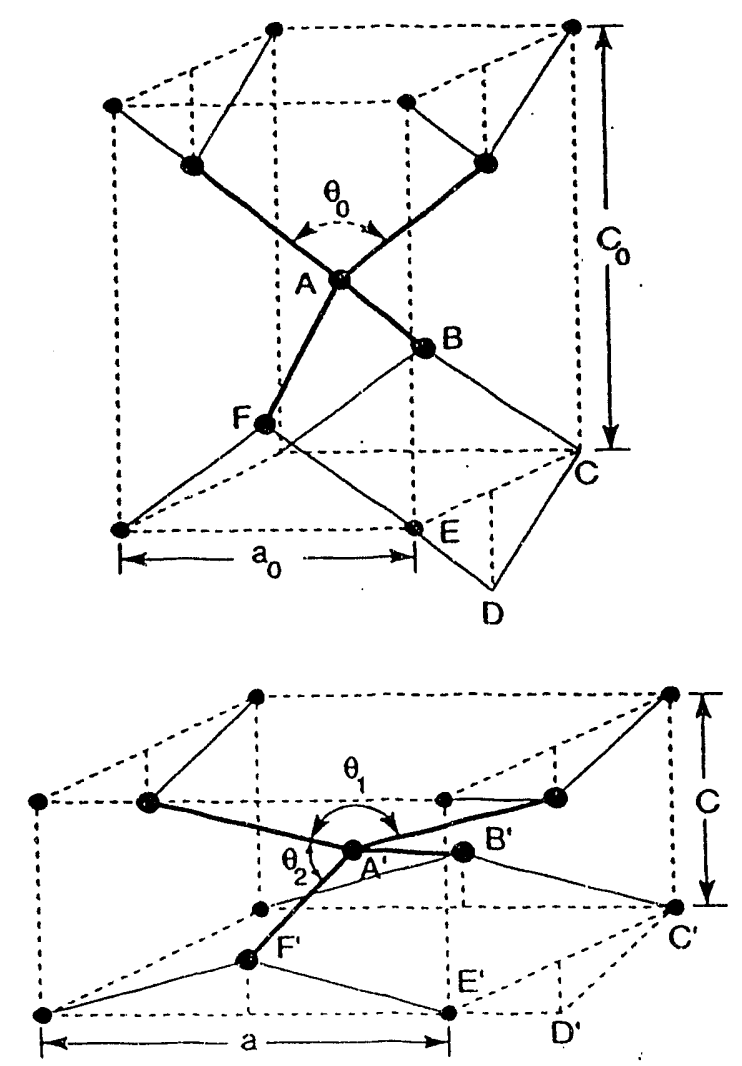

Figure 3 - Schematic relationships between the diamond and $\beta$-tin frameworks. For Sn: $\theta_{0}=$ $109.5^{\circ} ; \theta_{1}=149.5^{\circ} ; \theta_{2}=94^{\circ}$. Note that the "boat-ring", ABCDEF which has equal sides and equal angles in the diamond framework becomes the ring $A^{\prime} B^{\prime} C^{\prime} D^{\prime} E^{\prime} F^{\prime}$ which has equal sides but two sets of three angles in the $\beta$-tin structure. Also note that the next-nearest-neighbor distance, $c$, in the $\beta$-tin structure is $3.18 \AA$, compared with $3.01 \AA$ for the nearest-neighbor bond length.

transitions are commonly said to be "pressure-induced"; in reality the whole deformation tensor governs them, not just the isotropic compression scalar. Thus they are "deformation-induced"; or perhaps shear alone induces them.

Several of the other III-V compounds transform to the rock-salt, instead of the $\beta$-tin, structure. This can also happen through shearing, but will not be discussed here because it is not as straightforward as the $\beta$-tin case.

As Musgrave and Pople (1962) have pointed out, the $\beta$-tin framework can be reached by compressing the diamond framework along its cube edge while allowing it to expand laterally (Fig. 3). The elementary deformation consists of compressing a tetrahedral bonding unit along an axis that passes through its center and bisects the opposice edges; plus the topological constraint that "bond rings" such as $A B C D E F$ which becomes $A^{\prime} B^{\prime} C^{\prime} D^{\prime} E^{\prime} F$ ' are conserved; i.e., they remair, closed. In order for them to close while the nearest neighbor bond lengths remain equal, the ring symmetry must change from three-fold to two-fold. This occurs more readily than symmetry preserving triaxial compression because the bending force constants are substantially smaller than the stretching constants.

For tin itself, the fractional changes in the crystallographic parameters are given at the top of Table I. Since the contraction of the $c$-axis is nearly twice the expansion of the a-axes, the 
Table 1

Per Cent Changes During the Transformation of the Diamond to the $\theta$-Tin Framework (Elements and III-V Compounds)

\begin{tabular}{|c|c|c|c|c|c|}
\hline & \multicolumn{2}{|c|}{$\begin{array}{c}\text { Lattice Parameter (A) } \\
\text {-tetragonal cells- }\end{array}$} & \multirow{2}{*}{$\frac{\begin{array}{c}\text { Bond } \\
\text { Length }(A)\end{array}}{d}$} & \multirow{2}{*}{$\begin{array}{c}\begin{array}{c}\text { Bond } \\
\text { Angles }\end{array} \\
\theta \text { (deg.) }\end{array}$} & \multirow{2}{*}{$\frac{\begin{array}{c}\text { Cell } \\
\text { Volume }\end{array}}{\text { v }}$} \\
\hline & $\mathbf{a}$ & $c$ & & & \\
\hline Sn & +27.4 & -50.9 & +7.9 & $+37 ;-14$ & -20.3 \\
\hline Ge & +22.3 & -52.4 & +3.2 & $+35 ;-14$ & -28.8 \\
\hline Si & +22.0 & -52.4 & +3.4 & $+36 ;-14$ & -29.1 \\
\hline C & $(+22)$ & $(-51.9)$ & $(+3.2)$ & $(+36 ;-14)$ & $(-28.6)$ \\
\hline InSb & +20.9 & -51 & +6.8 & $+37 ;-14$ & -22 \\
\hline GaSb & +24.1 & -51.8 & +4.9 & $+36 ;-14$ & -25.8 \\
\hline AISb & +24.1 & -52.8 & +4.5 & $+37 ;-14$ & -27.6 \\
\hline InAs & +22.0 & -57.0 & +2.7 & $+39 ;-15$ & -36 \\
\hline GaP & +22.5 & -54.7 & +3.4 & $+38 ;-14$ & -32.1 \\
\hline
\end{tabular}

Table data references:

$\mathrm{Sn}, \mathrm{Ge}, \mathrm{Si}$ - Landolt-Bornste in Tables

C - Estimate of uniaxial transformation strain from 0 . Nielson, Phys. Rev., 34B, 5808 (1986).

InSb - R.E.Hanneman, M.D.Banus and H.C.Gatos, J.Phys.Chem.Solids, 25,293 (1964).

InAs - J.C.Jamieson, Science, 139, 752, 845 (1963)

AlSb - M.A.Baublitz and A.L.Ruoff, J.Appl.Phys., 53, 6179 (1982).

GaSb - C.Yu, I.L.Spain and E.F.Skelton, Sol.St.Comm., 25, 49 (1978).

GaP - A. L. Ruoff and M. A. Baublitz, Phys. Solids High Pressure, Ed. by Schilling and Shelton, North-Holland Publ.Co., p.81 (1981).

volume change is modest. A point that is partisularly noteworthy is that the fractional bondangle changes are much larger $(5-10 X)$ than the hond-length changes. Furthermore, the table indicates that ihe critical bond-angle change (and therefore the critical bond-angle) is essentially invariant for all eight of the known cases. Also, the bond length changes are small in all cases. Thus the crystallography as well as the mechanics indicates that shear predominantly induces the transition; not isotropic compression. It will be shown shortly that this is also consistent with the theory of chemical bonds.

Notice that the first nearest-neighbor distance in the $\beta$-tin structure is $3.01 \mathrm{~A}$. while the second nearest-neighbor distance is $3.18 \mathrm{~A}$. So the difference is only $5.6 \%$. This has led many authors to assert that the coordination number is 6 , rather than 4 , in $\beta$-Sn. However, conservation of orbital continuity requires that it be 4 as suggested by Fig. 3 .

It has become commonplace to discuss these semiconductor transitions in terms of energy vs. density ciagrams (Yin and Cohen, 1980), but Fig. 3 and the text above indicates that this obscures the nature of the change. The change is primarily one of shape; and only secondarily of specific volume. The same comment applies to other substances. For example, it applies to the transformations in silicates and phosphates which also involve covalent bonds. In such cases, volume change is not an adequate descriptor of either the structural, or the energetic, factors.

Little is known experimentally about the state of deformation at the start of the transformation. Typically, only the "pressure" is reported; and/or the "volume". But it is not clear whether the material still has cubic symmetry; and to what level of precision.

\section{BOND-BENDING CRITERION FOR TRANSFORMATION}

A simple criterion for shear metallization can be derived from Pauling's original (approximate) 
theory of the chemical bond (Glasstone, 1944). In the most simple version of this theory, the form of the wave-function for a hybrid $\mathrm{sp}^{3}$ orbital is (angular dependence only; the radial part is assumed to be unchanged by hybridization):

$$
\psi_{h}=1 / 2(1+3 \cos \theta)
$$

where $\theta$ is the angle with respect to the direction of the bond. The first term represents the s-part of the orbital while the second represents the p-part. The bond energy is proportional to the square of this; and the anti-bonding orbital has a similar form except that its energy decreases with increasing bond-angle. The energy difference, or gap, between the bonding and anti-bonding energies decreases toward zero as the bond angle increases from its initial value. The gap becomes zero when the bond angle becomes $148.5^{\circ}$ which is close to the $149.5^{\circ}$ observed for tin. The excellent agreement may be fortuitous, but the calculation illustrates the principle that there is a strong dependence of bond energy on bond angle, and that there is a critical angle at which the bonding becomes metallic. Numerical band-theory calculations are consistent with this (Chelikowsky, 1987).

Further support can be given to this geometric criterion by showing that it yields the correct energy condition. That is, by showing that the work done in changing the bond-angle equals the energy needed to close the energy gap.

Imagine $a$ bond of length, $b$ that is held in place at one end and acted on by a tangential force, $f$ at the other end. The force tends to change the bond angle, $\theta$, and is resisted by a boridbending force-constant, $\mathrm{k}_{\theta}(\mathrm{d}-\mathrm{cm})$. For a small change of the angle, the incremental work, $\mathrm{dW}$ done by the force is: $f b d \theta=k_{\theta} \theta d \theta$. Integration yields:

$$
W=K_{\theta}(\Delta \theta)^{2} / 2
$$

and since the observed value of $\Delta \theta$ is 0.7 radians, $W=k_{\theta} / 4$. This is to be compared with onehalf of the energy gap $k \theta$ has been defined in various ways, but most convenient is Harrison's (1980) definition: $k_{\theta}=\left(3 b^{3} / 8\right)\left(C_{11}-C_{12}\right)$ which relates it to the standard elastic constants, $C_{i j}$.

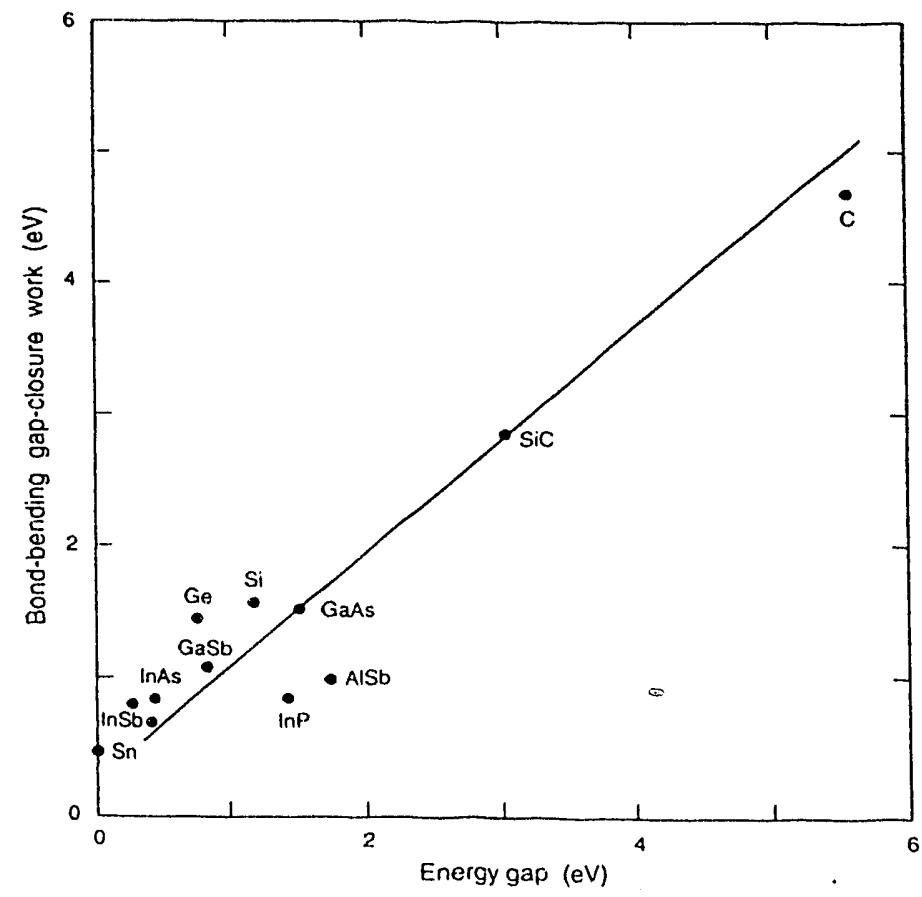

Figure 4 - Work required to bend bonds from their initial tetrahedral angles up to the critical transformation angle plotted against the average energy gap of the material. 
If the bond-bending hypothesis is valid the force constant should be related to the band-gap, and Fig. 4 shows that indeed it is. Thus, as the gap increases, so does the force constant, and the angle change needed to close the gap remains approximately constant.

\section{CONSEQUENCES OF METALLIZATION AND HYPERVELOCITY IMPACTS}

For states of uniaxial compression such as those experienced in the inertial confinement of strong shock waves, or in the confined static compression of indentations, bond-angle changes are accompanied by bond-length decreases, and metallization is induced by the combination. However, since the stretching force constants are much larger than the bending force constants (by a factor of $\sim 4$ ) they provide most of the resistance to the loading, and bending accommodates most of the deformation. This may account for the correlations that have been found between transformation pressures and indentation hardnesses (Gilman, 1992). An example of this correlation is shown in Fig. 5. This indicates quite strongly that there is a connection between flow under conditions of high deformation and electronic transitions in these materials. It should then be expected that hard materials that are impacted at hypervelocities will transform through delocalization of their bonding electrons into states that will flow more readily than might otherwise be expected.

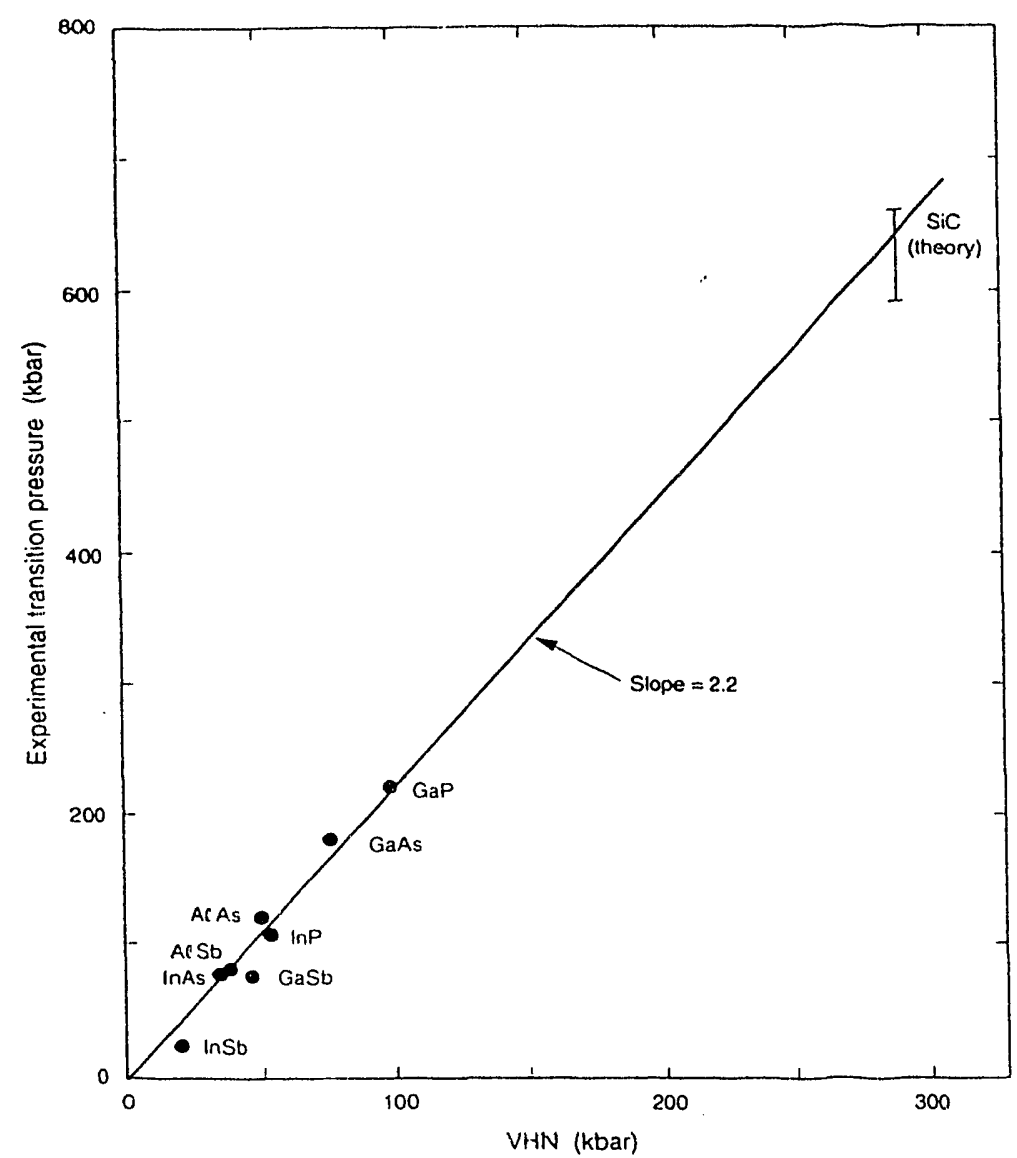

Figure 5 - Insulator-metal transition pressures correlated with Vickers hardness numbers (VHN). The correlation suggests that bond-bending plays an important role in both processes.

If it is accepted that shear can induce metallization, there are implications for many situations that do not appear to have been appreciated in the past. These include: point-contact diodes and transistors (Clarke et al.,1988), various allotropic transformations including those involving 
the d-shell bonding in metals, chemical reactivity by facilitating electron-transfers(Gilman, 1992), the core structures of dislocations and dislocation-dipoles, the mechanisms of machining and grinding, Mode III crack propagation, reversible compression-induced transitions in silicatelike frameworks, impacts and shock ironts, friction and wear phenomena, and indentations (Gilman, 1992).

In the last case, a dramatic photograph has been obtained by Pharr, Oliver, and Harding (1991). This shows material extruding out from under a diamond pyramid as it indents silicon. An interpretation is that the compression created by the indentation has metallized the silicon locally thereby allowing it to extrude like a metal. This observation has led to the proposal that this is a common phenomena when similar states of deformation are present.

A connection between impact yield stresses (Hugoniot elastic limits) for hard materials was demonstrated by the author previously (Gilman, 1970; 1975). The connection is illustrated by Fig. 6 . In the light of the evidence presented here for the $s p^{3}$ bonded semiconductors, it is natural to wonder whether impact has a large effect on the electronic (bonding) structures of hard compounds like $B_{4} C$. Does the Hugoniot elastic limit represent a phase transition? Or does it represent the stress needed to move low mobility dislocations? What is the role of shear deformation compared with that of isotropic compression? How might one answer these interesting questions?

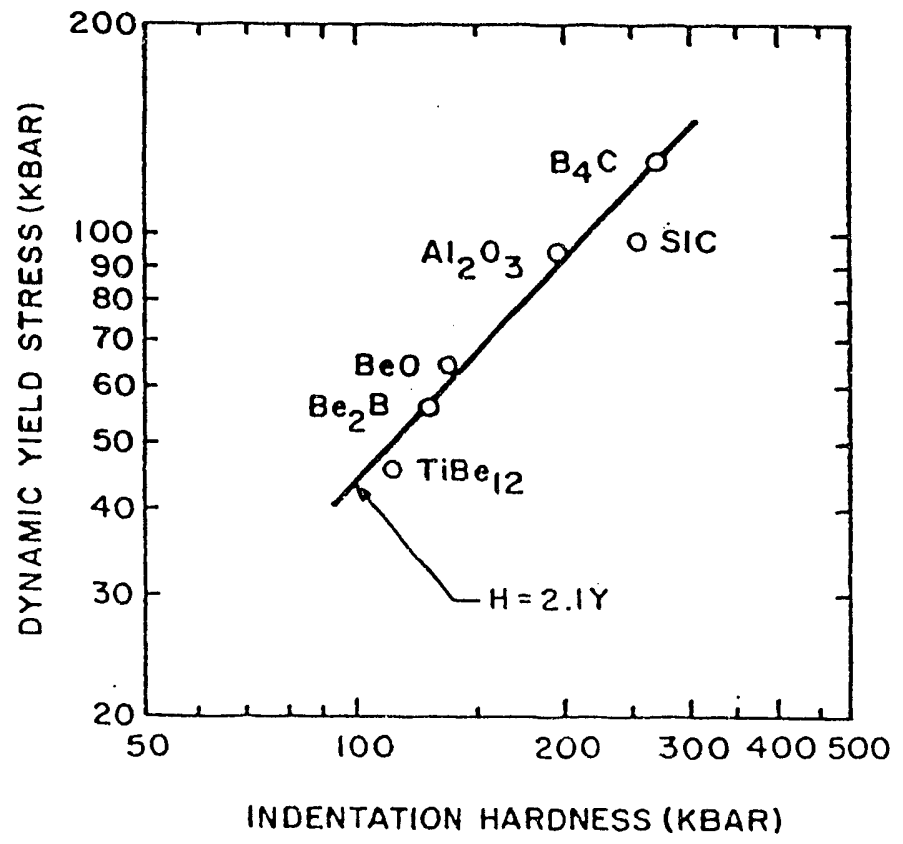

Figure 6 - Relationship of dynamic (explosive impact) yield stresses to static yield stresses (from indentation hardness) for various hard compounds. The ratio of these yield stresses to the elastic shear stiffnesses for these compounds is the same as for those of Figure 8. The data are from C. F. Cline.

Since all solids become metallic when compressed sufficiently, they will all metallize at sufficiently high impact velocities. So the question is not whether hypervelocity impact causes changes in the electronic structures of solids (or liquids); the question is when? And when does shear deformation facilitate the process?

The velocity needed to irarisform silicon, for example, in an impact with itself is about 1.3 $\mathrm{km} / \mathrm{sec}$. This assumes a critical strain of about 0.2 ; a bulk modulus of $2.24 \mathrm{Mb}$; and a specific gravity of 2.33. For $\mathrm{SiC}$ the critical impact velocity is about $1.6 \mathrm{~km} / \mathrm{sec}$. Thus the transformation can be expected under typical hypervelocity conditions. 


\section{INSULIZATION, THE INVERSE OF METALLIZATION}

For tensile states of strain in metals, metallization is not expected, but the inverse; that is "insulization", is. It is expected that insulization will be deformation dependent (i.e., dependent on both dilatation and shear). This would change the metallic bonding from a delocalized mode to a localized one. Therefore, it might well play a role in such phenomena as crack propagation in which large tensile strains exist near crack tips. It might also account for why the spalling of metals that is associated with strong reflected shock waves is often very localized. And it may account in part for the weakening effects of large concentrations of dislocations; as well as the hardening effects of dilatational dislocation dipoles in metals. Localization of the bonding would be expected to have a strong influence on the microscopic mechanisms of these various phenomena.

Bonding in most of the metals that are used to build engineering structures is associated with interactions of the d-type electrons $(3 d, 4 d$, and $5 d$ ). These interactions are responsible for the high cohesive energies, the high stiffnesses, and melting points of the structural metals. Ductility in these metals is associated with small (relatively) shear moduli. That is, with low values of the ratio of the shear to the bulk modulus (Gilman, Cunningham and Holt, 1990). This, in turn, is related to delocalization of the bonding electrons, particularly the d-electrons since the $s$ - and p-electrons contribute relatively little to either the bonding or the shear resistance. These comments apply to the pure metals; alloying may also play a crucial role.

One manifestation of the importance of the $d$-bonding is the fact that iron cleaves on its (100) planes rather than the mos close-packed (110) planes. Another direct manifestation is the role that it plays in determining dislocation mobility in the "hard metals"; carbides, borides, and nitrides (Gilman, 1970).

The d-orbitals terd to be more compact than the s- or p-orbitals so only their tip regions tend to overlap in transition metal crystals (Cottrell, 1988). The more the overlap the more the delocalization. Thus, if such a crystal is extended so the overlap is reduced, localization (and bond directionality) increases, and properties such as dislocation mobility tend to decrease, while crack tip localization tends to increase.

The standard localization parameter is called the "hopping Integral" which measures how fast electrons tend to hop from one atom to another (Cottrell, 1988). In other words how readily they move away from any particular locality. For the strongest d-bonds (called ddo-bonds), it has the following form:

$$
\text { hopping integral }=\beta=-2.4 w\left(r_{s} / b\right)^{5}
$$

where $w=d$-band width, $r_{s}=$ Wigner-Seitz radius, and $b=$ bond length. The inverse fifthpower dependence of $\beta$ indicates that small extensions can cause large increases in localization. For example, extending the bond length by $25 \%$ decreases the hopping integral by a factor of $\sim 3$.

Very large extensions of metals will convert them into insulators when the $s$ and p-electrons become localized. However, these extensions are so large that they will rarely be consequential in practice. However, localization will tend to increase shear stiffness relative to extensional stiffness, and this will change the overall mechanical response.

\section{POSSIBLE ROLE OF DELOCALIZATION IN DISLOCATION MOTION}

Hard materials may be divided into two hardness classes; intrinsic and extrinsic. The extrinsic ones are those in which dislocations move readily through perfect crystals of the base composition, but are inhibited by defects such as other dislocations, dipoles, impurities, grain 
boundaries, and precipitates. Most crystalline metals aid many ivnic cornpounds belong to this class. Intrinsic ones are trose in which dislocation mobilities are low elven in perfect crystals. Examples of these are semiconductors, interstitial metal compourids, and metallic glasses. In the intrinsic class, the behavior suggests that electronic structure plays an important, and intrinsis, role. Some of the lasts leading to this conclusion are:

a. the correlation between the thermal activation energy for dislocation motion and electronic energy gaps (Fig. 7) (Gilman, 1975).

b. the large ratio of the hardness number and/or the Hugoniol elastic limit to the elastic shear stiffness (Figs. 6 and 8) (Gilman, 1973).

c. the connection between the critical transiormation pressure and the hardness number (Fig. 5).

$d$. the influence of light and other elizctrical disturbances on hardness, and plastic flow, including surface effects (McColm, 1990$)$.

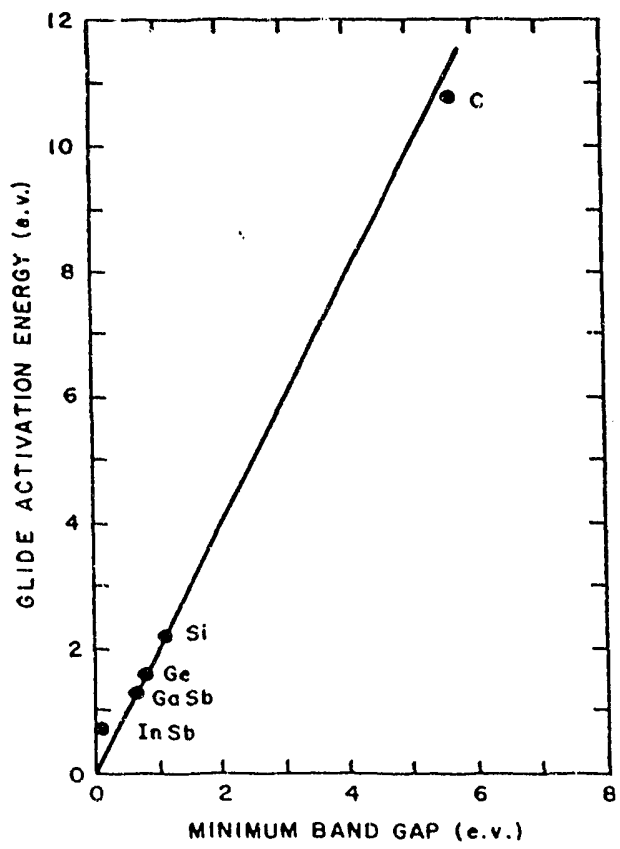

Figure 7 - Activation energy for the motion of dislocations versus the minimum energy gap for various covalently bonded crystals.

The connection between the mechanics and the electronics occurs through the bond-bending effects at the cores of dislocations and cracks. On the glide plane of a dislocation, the shear strain is a maximum at the center of the core and falls away to zero on both sides. At the center, if the spacing of the glide planes is $h$, and the Burgers displacement is $\delta$, the maximum shear strain is $\delta / 2 h$.

Then, taking semiconductors as an example, in the diamond framework $h=b$, and $\delta=1.63 \mathrm{~b}$, so the maximum strain is 0.82 and the bond-bending angle is the inverse tangent of this or $39.2^{\circ}$. This plus the tetrahedral angle $\left(109.5^{\circ}\right)$ yields $148.7^{\circ}$ which is the angle at which the energy gap closes. Closing the gap is equivalent to "breaking" the bond, and being an irreversible process, it limits the dislocation motion. Similarly, it is equivalent to the band-toband tunneling process that was proposed previously (Gilman, 1975) to account for low- 
temperature dislocation mobility. At higher temperatures, it is expected that phonons will assist the bond-bending process, thereby increasing dislocation mobility.

Impact loading causes countervailing effects which complicate interpretatinns. On one side, the high strain-rates minimize the time available for strain-relaxation through dislocation motion and multiplication. On the other side, high stresses tend to be present which delocalize bonding electrons and thereby enhance mobilities.

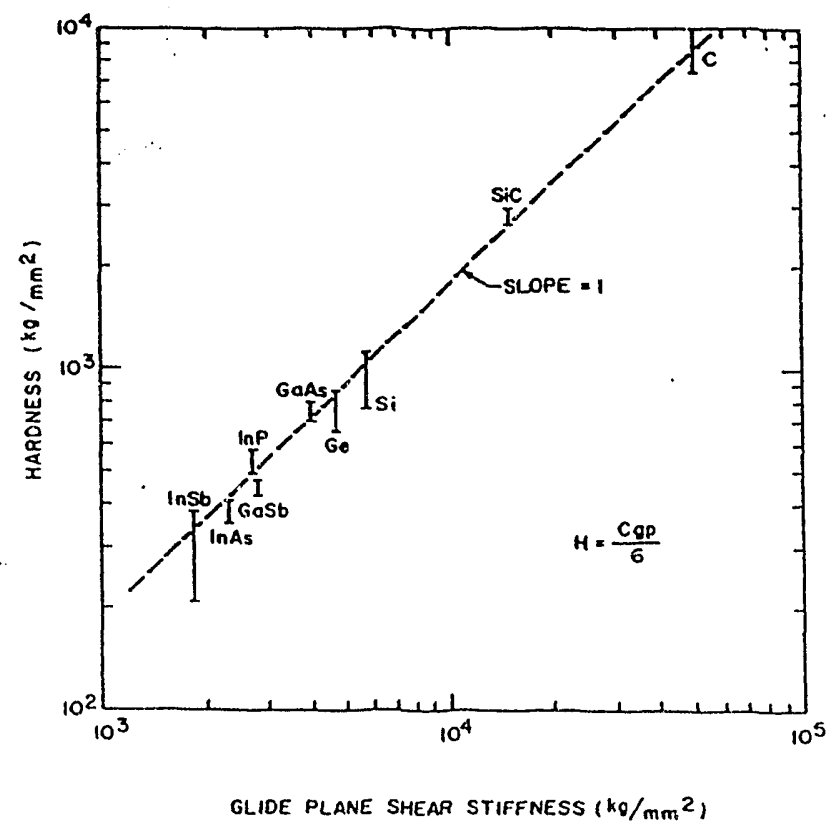

Figure 8 - Showing that the ratio of the Vickers hardnesses of a variety of tetrahedrally bonded crystals to their elastic shear stiffnesses is $1 / 6$. Thus the corresponding ratio for their shear flow stresses is $\simeq 1 / 18$.

\section{CONCLUSION}

Evidence has been presented showing that the large shear deformations that occur during impact can cause semiconductors to transform into metals. Thus materials that are normally quite brittle can become ductile. Their yield stresses can decrease dramatically. Criteria for this phenomenon are given that are derived from the theory of chemical bonding. The transformation occurs when covalent bonds are bent from their normal angles by a critical amount (for tetrahedral bonds, when the bond angle goes from $109.5^{\circ}$ to $149.5^{\circ}$ ). Compression must also be present.

Expansion causes the opposite of metallization; namely, insulization. That is localization of bonding electrons. This may be important in the behavior of expanded transition metals. Localization of the bonding d-electrons will modify the behavior of dislocations and cracks in these expanded metals. 


\section{REFERENCES}

Chelikowsky, J. R., Phys. Rev., 35B, 1174 (1987).

Clarke, D. R., Kroll, M. C., Kirchner, P. D., Cook, R. F. and Hockey, B. J., Phys.Rev.Lett., 60, 2156 (1988).

Cottrell, A. H. (1988), Introduction to the Modern Theory of Metals, Chapter 1 , Institute of Metals, London.

Edwards, P. P. and Sienko, M. J., Int. Rev. Phys. Chem., 3, 83 (1983).

Gilman, J. J., J. Appl. Phys., 11, 1664 (1970).

Gilman, J. J., in The Science of Hardness Testing and its Research Applications, Ed. by Westbrook and Conrad, Chapter 4, p.60, American Society for Metals, Metals Park, Ohio (1973).

Gilman, J. J., J. Appl. Phys., 46, 5110 (1975).

Gilman, J. J., Cunningham, B. J., and Holt, A. C., Mater. Sci. and Eng., A125, 39 (1990).

Gilman, J. J., "Detonarion via Metalli tation in Solids", Bull. Amer. Phys. Soc., 37, 361 (1992).

Gilman, J. J., J. Mater. Res., 7, 535 (1992).

Glasstone, S. S., Theoretical Chemistry, p.97ff, Van Nostrand Company, New York (1944).

Gupta, M. C. and Ruoff, A. L., J. Appl. Phys., 51. 1072 (1980).

Harrison, W. A., Electronic Structure and Properties of Solids, p. 193ff, W. H. Freeman, San Francisco (1980).

Herzfeld, K. F., Phys. Rev., 29, 701 (1927).

Mciolm, I. J., Ceramis Hardness, p. 135, Plenum Press, New York (1990).

Mott, N. F., Proc. Phys. Soc., 62A, 416 (1949).

Musgrave, M. P. J. and Pople, J. A., J.Phys.Chem.Solids, 23, 321 (1962).

Pharr, G. M., Oliver, W. C., and Harding, J. Mater. Res., $\underline{6}, 1129$ (1991).

Yin, M. T. and Cohen, M. L., Phys.Rev. Lett., 45, 1004 (1980). 

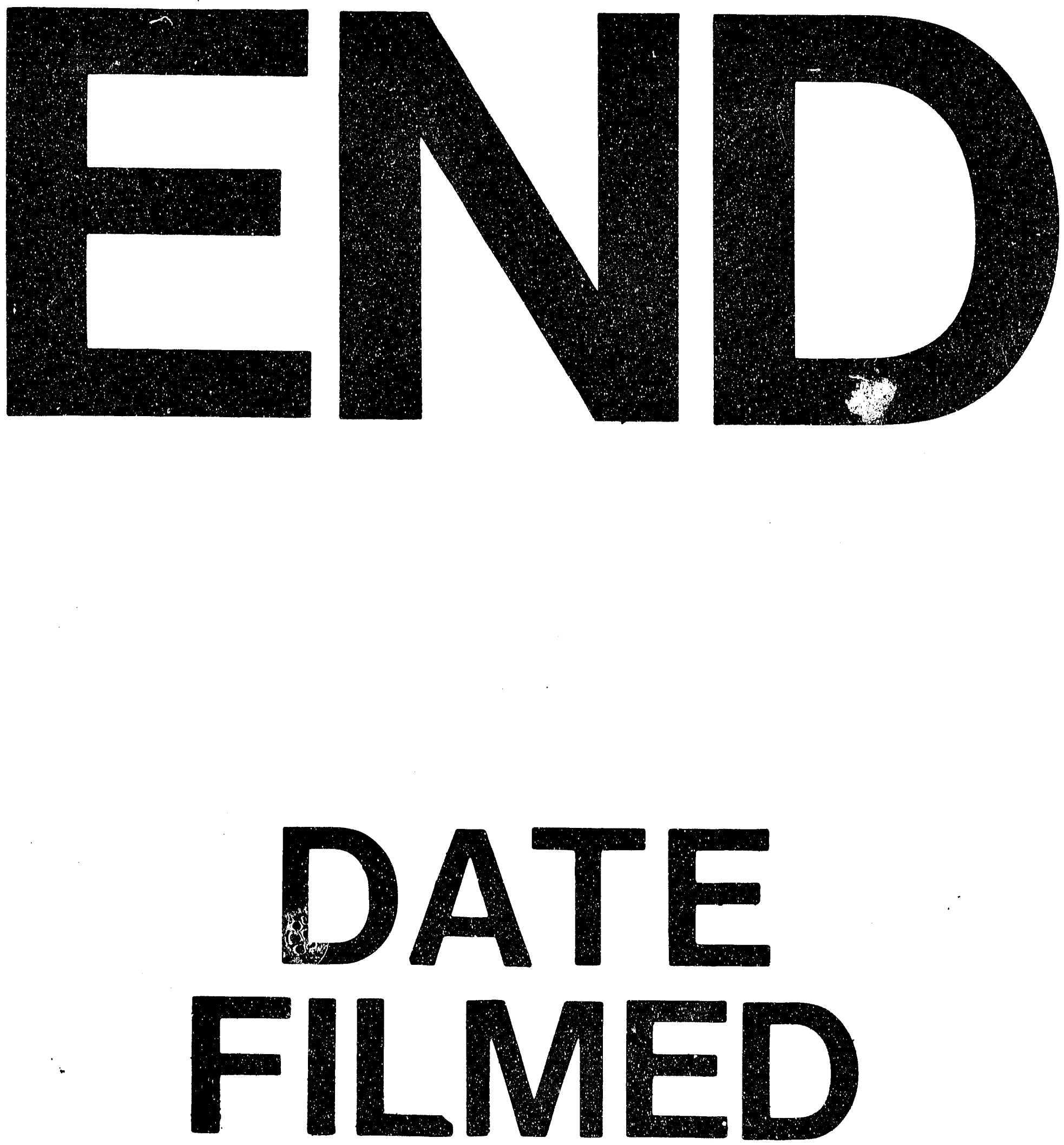

1
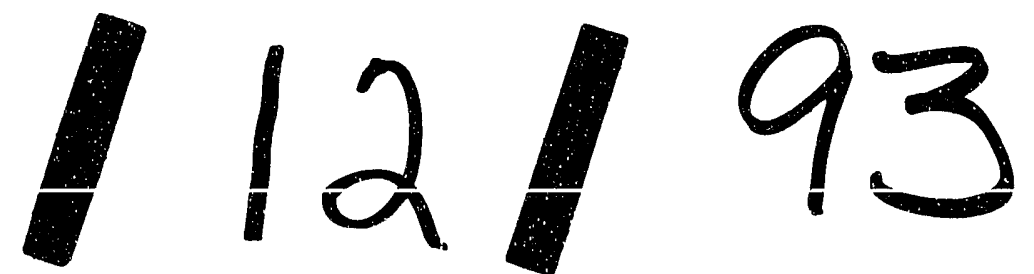
